\title{
Atherosclerotic Plaque Delamination: Experiments and 2D Finite Element Model to Simulate Plaque Peeling in Two Strains of Transgenic Mice
}

Bilal Merei , Pierre Badel, Lindsey Davis, Michael A. Sutton, Stéphane Avril*, Susan Lessner

Bilal Merei: merei@emse.fr

- SaInBioSE, INSERM, U1057, Mines Saint-Etienne, F-42023 Saint Etienne, France

- Department of Biomedical Engineering, University of South Carolina, Columbia, South Carolina 29208, USA

Pierre Badel: badel@emse.fr

- SaInBioSE, INSERM, U1057, Mines Saint-Etienne, F-42023 Saint Etienne, France

Lindsey Davis: davis79@email.sc.edu

- Department of Biomedical Engineering, University of South Carolina, Columbia, South Carolina 29208, USA

Mickael Sutton : sutton@cec.sc.edu

- Department of Mechanical Engineering, University of South Carolina, Columbia, South Carolina 29208, USA

Stéphane Avril: avril@emse.fr

- SaInBioSE, INSERM, U1057, Mines Saint-Etienne, F-42023 Saint Etienne, France

Susan Lessner: lessner@uscmed.sc.edu

- Department of Cell Biology and Anatomy, University of South Carolina School of Medicine, Columbia, South Carolina 29208, USA

* corresponding author 


\begin{abstract}
Finite element analyses using cohesive zone models (CZM) can be used to predict the fracture of atherosclerotic plaques but this requires setting appropriate values of the model parameters. In this study, material parameters of a CZM were identified for the first time on two groups of mice $\left(\mathrm{ApoE}^{-/-}\right.$and $\left.\mathrm{ApoE}^{-/-} \mathrm{Col}^{-/-}\right)$using the measured force-displacement curves acquired during delamination tests. To this end, a $2 D$ finite-element model of each plaque was solved using an explicit integration scheme. Each constituent of the plaque was modeled with a neo-Hookean strain energy density function and a CZM was used for the interface. The model parameters were calibrated by minimizing the quadratic deviation between the experimental force displacement curves and the model predictions. The elastic parameter of the plaque and the CZM interfacial parameter were successfully identified for a cohort of 11 mice. The results revealed that only the elastic parameter was significantly different between the two groups, ApoE ${ }^{-/-}$Col $^{-/-}$plaques being less stiff than ApoE ${ }^{-/}$plaques. Finally, this study demonstrated that a simple 2D finite element model with cohesive elements can reproduce fairly well the plaque peeling global response. Future work will focus on understanding the main biological determinants of regional and inter-individual variations of the material parameters used in the model.
\end{abstract}

Keywords: Cohesive zone model, plaque delamination, explicit scheme, inverse approach, parameter identification, collagen VIII 


\section{Introduction}

Atherosclerotic plaque rupture is a major cause of myocardial infarction, coronary thrombosis and stroke. Cardiovascular diseases resulting from atherosclerosis are the leading cause of mortality in both developed and developing countries. Three-fourths of myocardial infarctions are caused by the rupture of atherosclerotic plaques, affecting about 1.1 million people in the US annually, with a fatality rate of $40 \%$; 220,000 of these deaths occur without hospitalization ${ }^{1}$. Thus, a better understanding of this disease is needed to develop effective approaches for treatment and intervention. Experimentally, several studies have focused on developing experimental protocols to quantify the adhesive strength of the bond between two biological materials ${ }^{2-6}$. To better understand the plaque delamination process, Wang et al. $2011^{7}$ developed and applied a methodology to quantify the adhesive strength between the atherosclerotic plaque and the underlying vascular wall. The method was applied to the apolipoprotein E knockout $\left(\operatorname{apoE}^{-/}\right)$mouse model after 8 months on Western diet. The apoE-deficient mouse is an animal model frequently used in atherosclerosis research due to the development of plaques of similar type and distribution as in humans ${ }^{8,9}$ and mice lacking apoE $\left(\mathrm{ApoE}^{-/}\right)$provided the first practical animal model of hyperlipidemia and atherosclerosis ${ }^{10}$. The study by Wang et al. used the local energy release rate, $\mathrm{G}$, as a quantifiable metric for direct comparison of plaque separation strengths.

On the computational side, cohesive zone models (CZM) have been applied to biological tissues to better understand a number of medical problems that involve separation of tissue layers. The cohesive zone is defined as the infinitesimally thin layer in which initialization and coalescence of micro-cracks are lumped into a discrete surface, based on the elasto-plastic fracture theory of metals ${ }^{11,12}$, and on the quasi-brittle fracture theory of concrete ${ }^{13}$. This approach is used to model 
the delamination or separation between layers. To understand some medical problems where fractures or separations between layers occur, the CZM has been used in modeling soft biological tissues $^{14-18}$ and bones ${ }^{19-22}$. These studies used CZM with traction-separation cohesive laws. None of these studies used experimental data obtained from direct mechanical experiments to identify both cohesive and material parameters at the same time. In the study presented here, a 2D numerical finite element model was developed to identify material parameters and cohesive parameters based on experimental data. The method we present could be applied to any medical problem where separation between layers occurs, such as arterial dissection or atherosclerotic plaque delamination. For soft biological tissues, Ferrara et al. 2010 used CZM to study the dissection properties of individual arterial tissues ${ }^{16}$. Gasser et al. 2006 used the CZM technique to model the propagation of arterial dissections using an explicit scheme ${ }^{14}$. In their study, they defined the dissection as a gradual process in which cohesive traction resists separation between two material surfaces. The presence of collagen in arterial layers motivated the use of this cohesive concept. A recent numerical study by Leng et al. 2015 also used CZM finite element analyses with an implicit resolution scheme to simulate atherosclerotic plaque delamination in ApoE knockout mouse abdominal aorta specimens, placing the cohesive zone along the plaquemedia interface where delamination occurs ${ }^{23}$. The simulation predictions of force-displacement curves for the simulated cycles were found to match reasonably well with the experimental data, especially for the plaque deformation phase, but differences were still observed during the separation phase and the unloading phase. Leng et al explained these differences by the fact that many parameters used in the model were not directly calculated but taken from existing values in the literature. They also considered that the use of the Holzapfel-Gasser-Ogden model (HGO) for the plaque could be the reason for these differences. 
In summary, an experimental protocol was developed by Wang et al. to study atherosclerotic plaque delamination as a fracture mechanics problem by quantifying the energy release rates, but few studies have used CZM to study this problem. Those that did generally did not consider an explicit resolution approach, and many parameter values were assumed due to a lack of geometrical data.

In the current study, we focused on developing a 2D finite element modeling and simulation approach, using an inverse method, to identify material and cohesive parameters based on experimental delamination tests between atherosclerotic plaque and the underlying vascular wall in Type VIII collagen-deficient and non-deficient (control) apoE knockout (ApoE $\left.{ }^{-/}\right)$mice. In this particular problem, an explicit dynamic method of resolution was used (Abaqus 6.13-1 Explicit). The collagen Type VIII, from the short-chain non-fibrillar collagen family, is present in small amounts in normal arteries. After injury and during development of atherosclerosis in experimental animals and humans, the synthesis of type VIII collagen is dramatically increased 24, 25 . Thus, comparison of experimental plaque delamination data from mice belonging to a control group $\left(\mathrm{ApoE}^{-/-}\right.$) and from a collagen VIII deficient group (ApoE ${ }^{-/-} \mathrm{Col}^{-/-}$) presented an interesting test case to develop the FE model. 


\section{Materials and methods}

\subsection{Experimental protocol}

Four C57B16 congenic ApoE ${ }^{-/-}$mice and seven $\mathrm{ApoE}^{-/-} \mathrm{Col}^{-/-}$mice were fed with a high-fat (40\% of total calories) diet during six months to develop advanced aortic atherosclerotic plaques. Mice were euthanized by carbon dioxide asphyxiation and perfused with heparinized saline at physiological pressure for five minutes. Mouse carcasses were firmly attached to a plate using adhesive tape. The aorta was opened longitudinally to visualize the atherosclerotic plaques. The adhesion strength between the atherosclerotic plaque and the internal elastic lamina (IEL) was measured with cyclic peeling experiments, based on a previously published protocol ${ }^{7,26}$. A Bose Electroforce 3200 Test Instrument was used to measure the force required for plaque delamination, and a stereomicroscope equipped with a CCD camera was used to capture images of the process. The Bose Test Instrument had two grips. One grip clamped the plate which held the mouse carcass with exposed aorta, and the other grip was attached to microclamps that held the tip of the plaque, after creating an initial notch to initiate delamination of the plaque.

Figure 5(a) shows a schematic of the experimental test setup. The Bose Electroforce 3200 Test Instrument applied controlled displacements to produce incremental delamination of the plaque, and the CCD camera acquired images of the newly exposed area underneath the plaque. Consecutive cycles were run with increasing total displacement until the plaque completely separated from the vessel wall. Figure 5(b) shows a schematic of the delamination process in an enlarged side view. Table 4 shows the total number of mice tested from each group (ApoE ${ }^{-/-}$and $\left.\mathrm{ApoE}^{-/-} \mathrm{Col}^{-/-}\right)$, with the number of plaques tested $\left(\mathrm{P}_{\mathrm{i}}\right.$ : where "i" is the index referring to the number of the plaque tested from the same mouse) and the total number of loading cycles obtained from each plaque. 


\subsection{Delamination Test and Data Acquisition}

\subsubsection{Determination of the fracture energy from each delamination cycle $\Delta E$}

Figure 6 shows an example of a force-displacement curve obtained during delamination. The curve is composed of three parts. The first part shows the initial ramp of the load versus displacement curve. This section is not part of the separation phase but represents the energy associated with deformation of the plaque before the event of separation. The first slope discontinuity of the curve represents the beginning of the delamination process that occurs when the measured load reaches a first maximum and drops. The second part of the curve is jagged or serrated; this region corresponds to the delamination process. The third part represents the unloading phase. The area of the region surrounded by the curve, represented in Figure 6, is the energy dissipated throughout one delamination cycle and it is denoted $\Delta \mathrm{E}$.

\subsubsection{Determination of exposed area $\triangle A$}

The area exposed at the plaque-IEL interface during one delamination cycle, $\Delta \mathrm{A}$, is

measured using Image ${ }^{27}$ by determining the area before delamination, $\mathrm{A}_{\mathrm{i}}$, and the area after delamination, $\mathrm{A}_{\mathrm{f}}$. To make this measurement, we applied diluted black marking tissue dye onto the surface of the plaque and onto its surrounding area before sequential delamination cycles. Pictures were taken before and after each cycle. At the end of the cycle the newly exposed area was white (or lighter than the surrounding area). The difference in colors was used to segment the newly exposed region and to measure its area $\Delta \mathrm{A}$ as defined in Eq. (1).

$$
\Delta \mathrm{A}=\mathrm{A}_{\mathrm{f}}-\mathrm{A}_{\mathrm{i}}
$$

Three independent reviewers measured $\Delta \mathrm{A}$ for each cycle of delamination. Area measurements that agreed within $10 \%$ between reviewers were averaged to determine the final value of $\Delta \mathrm{A}$ for 
each cycle. Figure 7 shows a sample where the white area (newly exposed region) has been delimited by a yellow line.

\subsubsection{Calculation of $G$ (energy release rate)}

The energy release rate, $\mathrm{G}(\mathrm{N} / \mathrm{mm})$, is a measure of adhesion strength and is calculated by dividing the energy released during delamination, $\Delta \mathrm{E}$, by the area exposed during the same delamination, $\Delta \mathrm{A}$ as shown in Eq. (2):

$$
G=\Delta \mathrm{E} / \Delta \mathrm{A}
$$

\subsubsection{Statistical analysis}

A Shapiro-Wilk test was used to test the normality of the distributions of G values. For normally distributed data, a t-test was performed to test for differences between the two genotypes and for non-normally distributed data, a Mann-Whitney nonparametric test was used to compare the median values between the $\mathrm{ApoE}^{-/-}$mice and the $\mathrm{ApoE}^{-/-} \mathrm{Col}^{-/-}$mice.

\subsection{Finite-Element model}

\subsubsection{Abaqus Explicit}

The explicit solver of the ABAQUS ${ }^{\circledR}$ software $^{27}$ was used in our simulations. An explicit solver in finite-element analyses uses an explicit time integration scheme to solve dynamic problems or quasi-static nonlinear problems. The explicit solver is particularly suitable for highly nonlinear problems as is the case here with contact and fracture issues ${ }^{28,29}$.

\subsubsection{Geometry}


Figure 8(a) shows a representation of the geometrical parameters used to create the 2D finite element model for our simulations. Some of these values could be measured by reference to images and experimental data, and others could not be measured. This was especially true of geometric parameters related to the aorta (media), such as the total length, the total width and the thickness. Therefore, we referred to values measured in other studies of similar problems and we assumed that these values could be applied in our simulations. The medial width $\left(\mathrm{W}_{\mathrm{m}}\right)$ was reported for $\mathrm{ApoE}^{-/-}$mice in the study of Gregersen et al.2007 30 to be in the range of $2 \mathrm{~mm}$. Medial height (or thickness, $\mathrm{H}_{\mathrm{m}}$ ) was also determined by the same authors to be in the range of 0.08 to $0.16 \mathrm{~mm}$. In our simulations, $\mathrm{H}_{\mathrm{m}}$ was set equal to $0.15 \mathrm{~mm}$. The total length of the aorta (media) could not be identified using the experimental pictures, so we assumed that $\mathrm{L}_{\mathrm{m}}$ was three times greater than the plaque length. A plate was added under the aorta with a frictionless contact to avoid displacement in the negative y-direction, as in the experiments. The total length of the plate was set equal to the length of the aorta.

\subsubsection{Plaque length $\left(\mathrm{L}_{\mathrm{p}}\right)$ measurement}

The plaque length was estimated using histological images. After total detachment of the plaque from the aorta, the plaque was kept for histology studies. The plaque was embedded vertically and cross sections of $5 \mu \mathrm{m}$ were made. Five sections were collected, then five sections were skipped, and this action was repeated until the entire plaque had been sectioned. The five collected sections represent a group. Each histological image was representative of these five sections forming a group. Thus, each image represented a plaque length of $25 \mu \mathrm{m}$. Adjacent groups were separated by another $25 \mu \mathrm{m}$ of sectioned length. Therefore, if there were ten histological images for a particular plaque the estimated length would be $500 \mathrm{um}$. $\mathrm{L}_{\mathrm{p}}$ values for 
each plaque are shown in Table 5. Note here that this calculated length was underestimated since some tissue shrinkage occurs upon fixation and embedding.

\subsubsection{Plaque height $\left(\mathrm{H}_{\mathrm{p}}\right)$ measurement}

The plaque height was also calculated using histological images. Assuming that the middle of the plaque has the largest height, the height of the middle section was measured and considered to be the maximum height of the plaque. Values are reported in Table $5 . \mathrm{H}_{\mathrm{p}}$ and $\mathrm{L}_{\mathrm{p}}$ were underestimated using this approach, since there was some tissue shrinkage during fixation and embedding.

\subsubsection{Plaque width $\left(\mathrm{W}_{\mathrm{p}}\right)$ measurement}

Assuming that the plaque width is the same along the length of the plaque, the plaque width was measured using the CCD camera images recorded during experiments. The values are reported in Table 5.

\subsubsection{Boundary Conditions}

Experimentally, the lower face of the aorta was free, since the vessel was secured across its width only with micro-pins placed a few millimeters above and below the plaque. These micro-pins are represented in the 2D model as fixed contact points between the aorta and the underlying plate at the left and right edges of the media. The underlying plate was added in contact with the aorta to avoid any displacement in the (-y) direction. Figure 8(b) shows a model with a thick plaque $(0.4 \mathrm{~mm})$, the aorta, and the cohesive layer as an extension of the notch of 1 $\mathrm{mm}$ created between the plaque and the underlying aorta.

Figure 9 shows four pictures at four different times of the simulation. It shows how the boundary conditions were assigned. The simulations were run in 2 steps. In the first step, a vertical 
displacement of $1 \mathrm{~mm}$ was applied on the master node to move the tip of the plaque to a vertical position, allowing at the same time free horizontal displacement and free rotation (Figure 5-a). Once the vertical displacement of $1 \mathrm{~mm}$ was reached (Figure 5-b), a horizontal displacement was applied in the dissection direction (Figure 5-c). For each sample, the horizontal displacement was set equal to the value applied in the respective experiment. This step simulated the plaque delamination stage where the data (force-displacement curves) were collected (Figure 5-d).

\subsubsection{Mesh size}

The geometry was meshed using plane strain quadrilateral elements. The cohesive zone was meshed using only quadrilateral structured elements of cohesive type.

After trying different mesh sizes for the plaque and media $(0.01,0.025,0.05 \mathrm{~mm})$, it was found that a mesh size set equal to $0.025 \mathrm{~mm}$ gives acceptable results within a reasonable computational time (less than $2.8 \%$ error when compared with the $0.01 \mathrm{~mm}$ mesh). The mesh size for the plate underneath the plaque was larger since this zone was kept rigid in this problem.

\subsubsection{Material model}

\subsubsection{Necrotic core}

The necrotic core, which is not as clearly defined in mouse plaques as in human plaques, was treated as having the same material properties as the fibrous cap.

\subsubsection{Fibrous cap and underlying aorta}

The fibrous cap and the underlying layer were modelled using a Neo-Hookean model. The strain energy function for a Neo-Hookean model is represented by Eq. (3): 


$$
\Psi=C_{10}\left(\overline{I_{1}}-3\right)+\frac{1}{D_{1}}\left(J_{e l}-1\right)^{2}
$$

Where $\mathrm{C}_{10}$ is the shear modulus, $\overline{I_{1}}$ is the first deviatoric strain invariant, $\mathrm{D}_{1}$ is the compressibility parameter, and $J_{e l}$ is the elastic volume ratio. Then, the first term of the equation represents the isotropic isochoric behavior and the second term represents the compressibility behavior. The compressibility modulus $\mathrm{D}_{1}$ was set equal to $1 \mathrm{MPa}^{-1}$. This corresponds to Poisson's ratios of 0.45 to 0.48 depending on the identified $\mathrm{C}_{10}$ values. These values of Poisson's ratio represent a moderately compressible behavior, which is usually admitted for atherosclerotic $\operatorname{arteries}^{33,34}$.

A Neo-Hookean model was used in several studies ${ }^{31,32}$ to represent the response of arterial tissues in the absence of collagen fiber recruitment. This model is widely used and accepted for small strains ${ }^{33,34}$. In addition, in this CZM problem, the elastic properties of the wall at larger strains are of secondary importance compared to the cohesive properties ${ }^{35}$.

The underlying plate was modeled as a linear elastic material (Young modulus: $1200 \mathrm{MPa}$, Poisson ratio: 0.44).

\subsubsection{Interface between the plaque and the aorta}

To represent the separation between the plaque and the underlying aorta, a bilinear traction separation cohesive law was used. Figure 10 depicts this law. It shows linear elastic loading $(\mathrm{OA})$, followed by linear softening $(\mathrm{AB})$. The normal maximum contact traction is reached at point $\mathrm{A}$ and denoted as $\mathrm{T}_{0}$. Separation starts at point $\mathrm{A}$ and ends at point $\mathrm{B}$ when the normal contact traction reaches zero. The area under the $\mathrm{OAB}$ curve is the energy released due to complete separation, which is termed the critical fracture energy per unit area. It is assumed that 
separation is cumulative and that any unloading/reloading cycle induces a purely elastic response along line OC.

The parameters of the bilinear traction separation cohesive law to be characterized are: $K_{\text {eff }}$ $(\mathrm{MPa} / \mathrm{mm}), \mathrm{T}_{0}(\mathrm{~N} / \mathrm{mm})$ and $\delta \mathrm{f}(\mathrm{mm})$ (since $\delta f$ and $\mathrm{T}_{0}$ are related - see Eq. (4) - only one of them will have to be identified).

\subsection{Parameter identification using an inverse method}

Teng et al. ${ }^{36}$ showed that, for $\mathrm{ApoE}^{-/-}$mice, $\mathrm{C}_{10}$ is 1.4 times larger in the fibrous cap (FC) than in the media and $\mathrm{C}_{10}$ is 1.6 times larger in the intraplaque haemorrhage/thrombus (IPH/T) than in the media. In the current study, $\mathrm{C}_{10}$ in the fibrous cap was set to twice the value of $\mathrm{C}_{10}$ in the media. This assumption is generalized in the rest of the simulations. Moreover, to avoid irrelevant solutions, bounds were defined for some of the unknown parameters.

$\mathrm{C}_{10}$ : values between 0.01 and $0.5 \mathrm{MPa}$

$\mathrm{T}_{0}$ : values between 0.05 and $0.2 \mathrm{MPa}$, which is consistent with values reported in the literature $e^{14}$.

Note that $\delta_{\mathrm{f}}$ and $\mathrm{T}_{0}$ are related to $\mathrm{G}$ by Eq. (4):

$$
G=\left(\frac{1}{2}\right) \times T_{0} \times \delta_{f}
$$

The values of $\mathrm{G}$ were calculated directly from the force displacement curves for each cycle. 
$\mathrm{K}_{\mathrm{nn}}$, the initial stiffness of the cohesive elements, does not represent a physically measurable quantity and is treated as a penalty parameter. The value of this penalty stiffness must be high enough to prevent interpenetration of the crack faces and to prevent the introduction of artificial compliance into the model by the cohesive elements ${ }^{37}$. However, an overly high value can lead to numerical problems. Therefore, the value considered in the simulations for $\mathrm{K}_{\mathrm{nn}}$ was $30 \mathrm{MPa} / \mathrm{mm}$.

In summary, two parameters of the model had to be identified from the experiments: the $\mathrm{C}_{10}$ elastic parameter of the plaque, and the $\mathrm{T}_{0}$ cohesive parameter. The inverse method consisted in finding the values of these two parameters that minimize the deviation between the experimental and the numerical force-displacement curves. An initial matrix containing all combinations of parameter values, $X_{\text {initial }}=\left[\mathrm{C}_{10}, \mathrm{~T}_{0}\right]$ is defined, and a cost vector was defined such that:

$$
\operatorname{cost}_{\text {vector }}(j)=F_{\text {sim }}(j)-F_{\text {exp }}(j)
$$

Where $F_{\operatorname{sim}}(j)$ is the force value predicted by the finite element model, $F_{\exp }(j)$ is the interpolated experimental force at the same displacement value, and $\mathrm{j}$ defines the index of the simulated point. Then the cost function value was calculated as in Eq. (6):

$$
\operatorname{cost}=\frac{\left[\operatorname{cost}_{\text {vector }}(j) x \cos t_{\text {vector }}^{T}(j)\right]}{{\overline{F_{\text {exp }}}}^{2}}
$$

Where ${\overline{F_{\text {exp }}}}^{2}$ represents the square of the average of the interpolated experimental force.

Finally, the minimum cost value was derived. Figure 11 shows an example of the pattern of the cost function for sample 173P1 with respect to the variations of $\mathrm{C}_{10}$ and $\mathrm{T}_{0}$. It appears that the cost function has a unique minimum for $\mathrm{T}_{0}=0.09$. 


\subsection{Energy balance}

At the end of each simulation, an energy balance study was performed to verify that the solutions obtained satisfy quasi-static mechanical equilibrium. The kinetic energy must be negligible compared to the strain energy to satisfy this criterion.

\section{Results}

\subsection{Experimental results}

Results showed that the majority of $G$ values were in the range [0.005-0.02] N/mm for both groups. The minimum value for both groups was $0.003 \mathrm{~N} / \mathrm{mm}$ and belonged to the ApoE ${ }^{-/}$ $\mathrm{Col}^{-/-}$group, and the highest value was $0.095 \mathrm{~N} / \mathrm{mm}$ and belonged to the same group. To compare the differences in parameters between both groups, a statistical analysis was applied. Table 6 summarizes the averages and the standard deviations obtained for the energy release rate (G), the slope of the linear part of the force-displacement curves, and the failure loads for each cycle. Average $\mathrm{G}$ values for both groups seemed to be similar with relatively large standard deviations for both groups $\left(0.015 \mathrm{~N} / \mathrm{mm}\right.$ for $\mathrm{ApoE}^{-/-}$group and vs $0.016 \mathrm{~N} / \mathrm{mm}$ for $\mathrm{ApoE}^{-/-} \mathrm{Col}^{-/-}$ group). G values for both genotypes were not normally distributed; therefore, a Mann-Whitney test was applied and showed that the values were not significantly different between groups.

\subsection{Numerical results}

After applying boundary conditions on the numerical model, the force-displacement curves obtained had the same shape as the experimental ones. Figure 12 shows a typical forcedisplacement curve obtained after simulation and after identifying the material parameters for one of the samples (173-P1). It shows that the curve was composed of three different segments 
as in experimental load-displacement curves (Figure 6). By comparing the changes in specimen geometry obtained after simulation (Figure 9) and the numerical curves, we could identify the mechanical process related to each part of the curve, as shown schematically in Figure 12.

The first segment (part 1) represents the deformation of the attached peeling arm of the plaque when the horizontal boundary condition was applied and before any separation occurred. The second segment (part 2) represents the separation between the plaque and the media layer. There were drops (a) and then increases (b) in force creating serrations as shown in Figure 12. Each drop in force represents a complete deletion of some cohesive elements because they had reached the maximum separation value. Then the force increased, which indicates that more cohesive elements were in the process of complete separation until they reached the maximum separation value and again created the release in force represented by the drops in Figure 12. The process of separation continued until the total horizontal displacement value was reached. The third segment (part 3) represents the unloading phase where an opposite horizontal displacement was applied on the attached arm of the plaque to take it back to the initial position. Figure 13 shows the results of the best-fit simulations with experimental curves for the first cycles from 4 different plaques from the $\mathrm{ApoE}^{-/-}$mouse group. It also shows the energy values during the simulations of the peeling test. In all cases the kinetic energy is negligible compared to the strain energy, which indicates that the solutions obtained satisfy quasi-static mechanical equilibrium. 
Figure 14 shows the best-fit parameters for the first cycles from 4 different plaques from the $\mathrm{ApoE}^{-/-} \mathrm{Col}^{-/-}$mouse group. It also shows the energy values during the peeling test calculated from the simulation, verifying that the kinetic energy is negligible compared to the strain energy.

The $\mathrm{T}_{0}$ (cohesive parameter) and $\mathrm{C}_{10}$ (material parameter) best fit values are reported in Figure 11. Figure 11 shows the average values of $\mathrm{G}, \mathrm{T}_{0}$ and $\mathrm{C}_{10}$ obtained for $\mathrm{ApoE}^{-/-}$samples and $\mathrm{ApoE}^{-/-} \mathrm{Col}^{-/-}$samples. Average values of $\mathrm{G}$ for the first group were higher than for the second. $\mathrm{T}_{0}$ values show a slight variation between the two groups. $\mathrm{C}_{10}$ average values between groups show an important difference, with the higher value for the $\mathrm{ApoE}^{-/-}$group.

A statistical test is needed to check for significant differences in the three mechanical parameters between the two mouse groups. However, due to limitations in the number of tested samples, this statistical test could not be applied. Therefore, we investigated the sample size needed to identify significant differences between groups for a T-test with $\alpha=0.05$. Alpha is defined as the Type I error probability for a two-sided test (the probability of false rejection of the null hypothesis). We found that 28 samples would have to be tested from each group with this amount of variation to determine whether there is a significant difference in $\mathrm{G}$ values between the two groups, while only 10 samples from each group would be needed to determine whether the differences were significant for $\mathrm{C}_{10}$ values between both groups. The test was not applied for $\mathrm{T}_{0}$ since the average values were similar. Fewer samples would be required to find significant differences between groups for $\mathrm{C}_{10}$ than for $\mathrm{G}$ ( or $\mathrm{T}_{0}$ ).

\section{Discussion}

\subsection{Discussion of experimental results}


Table 6 showed the average and standard deviation for $\mathrm{G}$ values obtained in both mouse genotypes used in our experiments. We can see that the average value of $\mathrm{G}$ for $\mathrm{ApoE}^{-/-} \mathrm{Col}^{-/-}$ mice was slightly higher than for $\mathrm{ApoE}^{-/-}$mice (0.016 vs $0.015 \mathrm{~N} / \mathrm{mm}$ ). However, the differences between the two genotypes were not significant. This result did not confirm the findings of Lopes et $\mathrm{al}^{38}$. These authors reported that deficiency of collagen VIII may affect the stability of the plaque by mediating fibrous cap formation. In fact, Lopes et al. $2013^{38}$ observed in their study that collagen VIII in the absence of apoE increases smooth muscle cell proliferation and migration. Consequently, formation of a thicker fibrous cap can be observed in the presence of collagen VIII, and a thinner cap is formed in its absence. A thinner fibrous cap has been previously associated with plaque instability in human patients ${ }^{1}$.

To check whether the duration of Western diet feeding could be a factor explaining these results (i.e., non-significant differences), control $\mathrm{ApoE}^{-/-}$mice were compared for two cases. In the first, mice were fed the Western diet for 8 months and in the second they were fed the same diet for 6 months. Wang et al. $2011^{7}$ quantified the rupture resistance of atherosclerotic plaques in $\mathrm{ApoE}^{-/-}$mice after 8 months on Western diet, using local delamination experiments and the corresponding local energy release rate $(G)$. In the present study, mice were tested after 6 months on Western diet. G values obtained after 8 months on Western diet, as reported in Wang et al. $2011^{7}$, varied between $0.005 \mathrm{~N} / \mathrm{mm}$ and $0.072 \mathrm{~N} / \mathrm{mm}$ with an average value of 0.024 and $\mathrm{SD}$ of 0.018 . Figure 16 shows the difference in $\mathrm{G}$ values between $\mathrm{ApoE}^{-/-}$mice fed Western diet for 6 months (present study) vs. 8 months (Wang, et al. 2011). The average G value in the 8month group $(0.024 \mathrm{~N} / \mathrm{mm})$ was higher than that for the 6-month group $(0.015 \mathrm{~N} / \mathrm{mm})$. This result may be due to the plaque fibrosis (collagen deposition) which would increase the energy required to cause delamination of the plaque (Wang, et al., 2013). Histological studies could determine 
more accurately the reason for the variation in $\mathrm{G}$ values with duration of Western diet feeding. Our study shows that the energy release rate is unaffected by the absence of type VIII collagen and suggests that other types of collagen may be responsible for the differences in adhesion strength previously reported, or simply that the sample size is not sufficiently large to prove the real role of collagen VIII deficiency.

\subsection{Discussion of numerical results}

In this work we identified for the first time material parameters and cohesive parameters for atherosclerotic plaques in two groups of $\mathrm{ApoE}^{-/-}$mice. To accomplish this, we developed an inverse method to calibrate a finite-element model against experimental force/displacement curves. These force/displacement curves were obtained with our specific delamination test ${ }^{7}$.

Leng et al. $2015^{23}$ presented finite element analyses of similar experimental tests and showed good agreement with experimental results, but they did not run an inverse analysis to identify material parameters. In the current study, to implement the inverse analysis, we had to reconsider completely the modeling approach of Leng et al. 2015 as it did not permit solution of the inverse problem for two reasons: 1 . Their model was 3D, which greatly increased the number of finite elements and consequently the computational cost. This approach is affordable for a small number of simulations but not for an iterative process of material parameter identification.

2. Their model used an exponential strain energy function (HGO) and the solution of the equations was based on an implicit scheme. These aspects make the convergence of the model very sensitive to the material and cohesive parameters. In the inverse analysis we needed the FE analysis to be robust in order to test many sets of material and cohesive model parameters during the minimization of the cost function. 
Another difference is that Leng et al. $2015^{23}$ did not use a frictionless contact between the aorta and the back wall of the mouse, but modeled the contact using springs, with parameters to be estimated. This again was required to permit convergence of the implicit solution scheme. In the current study we used a frictionless contact which was possible with the explicit solution.

In addition, we also observed that some convergence issues occurred at high $\mathrm{G}$ values with implicit resolution. The use of an explicit resolution scheme was able to give acceptable results for all samples despite the high $\mathrm{G}$ values and despite the presence of contacts between the support and the sample. Explicit schemes have been applied in several published studies where cohesive zone models were used to study dissection or fracture in soft biological tissues ${ }^{17,15,18,39}$. Recent studies that reviewed the advantages and limitations of using a cohesive zone model to study fracture showed that a precise determination of material parameters driving the tractionseparation relationship is essential for predictive CZM, which justifies the identification of parameters characterizing the traction-separation model and the surrounding material ${ }^{40,41,42,43}$.

The results shown in Figure 13 and Figure 14 utilize the best-fit parameters identified for a maximum agreement between experiment and simulation. The agreement was acceptable even though some differences remained, especially for the unloading phase. Serrations during the separation phase were always present in the experiments, but they were not reproduced with the implicit scheme ${ }^{23}$. Using the explicit scheme permitted reproducing these serrations during the separation phase. The occurrence of these serrations or the local drop of experimental force values may be explained by the fact that there are fibers bridging the plaque and the underlying artery, and sudden drops in force could be related to fiber breakage. This is not the case numerically, since the fibers were not taken into consideration in this model. Numerically, the serrations represent the propagation of delamination knowing that each drop in force means that 
the delamination has propagated a certain length, then the force increases to create another delamination. In summary, the explicit resolution is interesting as a means to simulate the serrations during the separation phase.

In our simulations we reduced the errors in the unloading phase, as is evident in some of the models (161-P1, 157-P1 and 173-P1), by optimizing the application of boundary conditions to represent exactly what was happening in the experiments. However, in some simulations, deviations from the experimental data in the unloading phase could still be observed. These discrepancies could be explained by the fact that a Neo-Hookean strain energy density function was used in our 2D simulations. Leng et al. $2015^{23}$ used a HGO strain energy density function for the material behavior and still had fitting issues for the unloading phase, which tends to confirm that improvements for the unloading phase have to be considered for future work. Moreover, the differences between simulations and experimental data may also be due to the assumptions made for some material parameters. Finally the Neo-Hookean strain energy density function works reasonably well for fitting the data, and this can be attributed to relatively low values of elastic strains preceding the beginning of delamination.

Values in the range [0.02-0.3] MPa were found for the $C_{10}$ parameter. Assoul et al. $2008{ }^{44}$ identified the elastic moduli of abdominal and thoracic aortas of $2 \mathrm{~mm}$ in diameter from adult Wistar rats and found values in the range [0.2-2.8] MPa, which is equivalent to $\mathrm{C}_{10}$ values in the range [0.035-0.5], since in general $\mathrm{C}_{10}=\mathrm{E} / 6$. The values obtained for $\mathrm{ApoE}^{-/-}$mice were in this range, but the values for $\mathrm{ApoE}^{-/-} \mathrm{Col}^{-/-}$mice were lower, which could be explained by the absence of collagen type VIII. This result is physiologically meaningful, as the deficiency of collagen VIII may affect collagen deposition and alter fibrous cap formation, as reported by Lopes et al. $2013^{38}$. Advanced atherosclerotic plaques typically contain a lipid pool and a 
fibrous cap. The lipid pool in the atherosclerotic plaque contains several constituents (phospholipids, cholesterol esters, cholesterol crystals and other lipids) ${ }^{45}$. Over time, liquid cholesterol esters may be transformed into a crystalline form, which could lead to a stiffer lipid pool ${ }^{46}$. This phenomenon might also explain the lower plaque stiffness in Col8 deficient mice due to the larger lipid fraction reported for this genotype. Few experimental data on the mechanical properties of lipid pools are available ${ }^{47}$. In our experiments, based on histological analysis, we observed that the lipid pool was always combined with other constituents. It was found using in vitro ultrasound elastography that the average elastic modulus of lipid was $81 \pm 40 \mathrm{kPa}$ for 9 human iliac arteries, but increased up to $1.0 \pm 0.63 \mathrm{MPa}$ when there was a mixture of smooth muscle cells and collagen fibers with the lipid ${ }^{48}$. Based on this study, we can justify merging the necrotic core and the fibrous cap into a single layer.

Our findings suggest that the adhesion strength of mouse atherosclerotic plaque is not affected by the absence of collagen VIII. We have also shown that the $\mathrm{ApoE}^{-/-} \mathrm{Col}^{-/-}$plaques are less stiff than the $\mathrm{ApoE}^{-/-}$plaques, which may be caused by the lack of type VIII collagen or by impaired migration of SMCs and resulting reduction in matrix deposition, as previously reported.

Notwithstanding these interesting conclusions, the experimental results have potential to reveal other interesting features of plaque biomechanics and future work will proceed along at least two main directions:

1. A hysteresis effect can be observed when unloading and reloading again between the first and the second cycle. The model (dashed red curve) does not reproduce this hysteresis as it does not account for viscoelasticity. However, the area of the hysteresis (energy dissipated due to viscoelastic effects) was about 10 times smaller than the area corresponding to the crack 
propagation. This observation supported our decision to neglect the viscoelastic effects in this study, where the objective was first to identify elastic and cohesive model parameters using only the first loading-unloading cycle. We still plan to identify viscoelastic parameters in the future by including several cycles in the identification, but this will require that we prove that the dissipation between two cycles is the result of viscoelastic effects only, as it may also be induced by damage in the tissue.

2. All the identified material parameters were identified using a plane strain model. We used the plane strain assumption as the width dimension (Wp) varied between 1 to 5 times the thickness $(\mathrm{H})$ of the plaque. However, for the sake of verification, we also compared the 2D plane strain model with a 2D plane stress model. Although none of these assumptions is perfect, the comparison showed that the dimensions of the sample in the third direction affect the delamination response significantly less than the material and cohesive model parameters that we wished to compare for the two strains of transgenic mice. But if we want to investigate other effects such as the curvature of the artery, the complex geometry of the plaque or the contribution of mode III in the delamination, for instance, refining the model would probably permit reaching a better agreement between experimental and numerical curves. Indeed, the model predictions obtained with the identified parameters have shown some discrepancies with regard to the experimental results. A 3D geometrical model reconstructed with the actual plaque geometry and an anisotropic nonlinear material model taking into account the regional histology would certainly provide improved accuracy.

The CZM technique seems to be a fairly good approach to gain a better understanding of delamination and shows a very good predictive capability in most cases, which is a convincing result for this proof-of concept study. The use of explicit scheme for simulations allowed us to 
capture the successive drops in load during the delamination process, but more studies have to be performed to correlate the numerical curves with the experiments by tracking the behavior of both experimental and numerical models in parallel to clearly identify the process leading to these sudden drops in force.

\section{Conclusion}

A cohesive zone model (CZM) approach was applied to simulate atherosclerotic plaque delamination experiments. Experiments were carried out on two mouse groups: ApoE ${ }^{-/-}$and $\mathrm{Apo}^{-}$ ${ }^{/-} \mathrm{Col}^{-/-}$. The experimental results showed that there are non-significant differences in G (critical energy release rate) values between the 2 groups. We then implemented a 2D finite element model in order to have a better understanding of the delamination process. An explicit resolution scheme was used to overcome limitations of implicit resolution methods applied previously to similar problems. An inverse method was used to identify two material parameters: one related to the interface (cohesive parameter) and one elastic parameter related to the plaque constitutive behavior. Results showed a very good agreement between experimental and numerical loaddisplacement curves after identification of the best-fit parameters. Average values obtained for both parameters revealed that only the elastic parameter could be considered different between the two groups. $\mathrm{Col}^{-/-} \mathrm{ApoE}^{-/-}$plaques were less stiff than $\mathrm{ApoE}^{-/-}$plaques, which may be attributed to the lack of type VIII collagen or to impaired migration of SMCs and the resulting decrease in matrix deposition ${ }^{38}$. Interfacial properties were non-significantly different. These results suggest that collagen VIII does not play a significant role in determining plaque adhesion strength to the underlying vessel wall. These trends deserve statistical confirmation with more experiments to be performed. Although the present study had led to these interesting conclusions, refining the model would probably permit a better agreement between experimental 
and numerical curves. To this end, we will consider in future studies a refinement of the model by creating 3D finite-element meshes taking into account fiber orientation, and a refinement of the CZM model including regional variations of interfacial properties for a more faithful prediction of the biomechanical response during delamination.

\section{Acknowledgements}

The authors would like to acknowledge financial support from the US National Science Foundation through grant number CMMI-1200358 and national center of scientific research CNRS Rhône-Alpes region. The authors would also like to acknowledge Dr. Michelle Bendeck, University of Toronto, for providing the $\mathrm{apoE}^{-/-} \mathrm{col}^{-/-}$mice used in the experimental study. The funding organizations played no role in the study design, data analysis, or manuscript preparation. 


\section{References}

1. Virmani, R., Narula, J., Leon, M. B. \& Willerson, J. T. The Vulnerable Atherosclerotic Plaque: Strategies for Diagnosis and Management. (John Wiley \& Sons, 2008).

2. Ahsan, T. \& Sah, R. L. Biomechanics of integrative cartilage repair. Osteoarthr. Cartil. OARS Osteoarthr. Res. Soc. 7, 29-40 (1999).

3. Maurice, D. M. \& Monroe, F. Cohesive strength of corneal lamellae. Exp. Eye Res. 50, 5963 (1990).

4. Dong, C. et al. Development of a device for measuring adherence of skin grafts to the wound surface. Ann. Biomed. Eng. 21, 51-55 (1993).

5. Roach, M. R. \& Song, S. H. Variations in strength of the porcine aorta as a function of location. Clin. Invest. Med. 17, 308-318 (1994).

6. Sommer, G., Gasser, T. C., Regitnig, P., Auer, M. \& Holzapfel, G. A. Dissection properties of the human aortic media: an experimental study. J. Biomech. Eng. 130, 021007 (2008).

7. Wang, Y., Ning, J., Johnson, J. A., Sutton, M. A. \& Lessner, S. M. Development of a quantitative mechanical test of atherosclerotic plaque stability. J. Biomech. 44, 2439-2445 (2011).

8. Zhang, S. H., Reddick, R. L., Piedrahita, J. A. \& Maeda, N. Spontaneous hypercholesterolemia and arterial lesions in mice lacking apolipoprotein E. Science 258, 468-471 (1992).

9. Nakashima, Y., Plump, A. S., Raines, E. W., Breslow, J. L. \& Ross, R. ApoE-deficient mice develop lesions of all phases of atherosclerosis throughout the arterial tree. Arterioscler. Thromb. Vasc. Biol. 14, 133-140 (1994). 
10. Pendse, A. A., Arbones-Mainar, J. M., Johnson, L. A., Altenburg, M. K. \& Maeda, N. Apolipoprotein E knock-out and knock-in mice: atherosclerosis, metabolic syndrome, and beyond. J. Lipid Res. 50, S178-S182 (2008).

11. Dugdale, D. Yielding of steel sheets con taining slits. J. Mech. Phys. Solids 8, 100-104 (1960).

12. Barenblatt, G. . The mathematical theory of equilibrium cracks in brittle fracture. Adv. Appl. Mech. 7 55-129 (1962).

13. Hillerborg, A., Modéer, M. \& Petersson, P.-E. Analysis of crack formation and crack growth in concrete by means of fracture mechanics and finite elements. Cem. Concr. Res. 6, 773781 (1976).

14. Gasser, T. C. \& Holzapfel, G. A. Modeling the propagation of arterial dissection. Eur. J. Mech. - ASolids 25, 617-633 (2006).

15. Gasser, T. C. \& Holzapfel, G. A. Geometrically non-linear and consistently linearized embedded strong discontinuity models for 3D problems with an application to the dissection analysis of soft biological tissues. Comput. Methods Appl. Mech. Eng. 192, 5059-5098 (2003).

16. Ferrara, A. \& Pandolfi, A. Numerical modelling of fracture in human arteries. Comput. Methods Biomech. Biomed. Engin. 11, 553-567 (2008).

17. Ferrara, A. \& Pandolfi, A. A numerical study of arterial media dissection processes. Int. J. Fract. 166, 21-33 (2010).

18. Caballero, A. \& Molinari, J. F. Finite element simulations of kidney stones fragmentation by direct impact: Tool geometry and multiple impacts. Int. J. Eng. Sci. 48, 253-264 (2010). 
19. An, B., Zhao, X., Arola, D. \& Zhang, D. Fracture analysis for biological materials with an expanded cohesive zone model. J. Biomech. 47, 2244-2248 (2014).

20. Ural, A. Prediction of Colles' fracture load in human radius using cohesive finite element modeling. J. Biomech. 42, 22-28 (2009).

21. Ural, A. \& Vashishth, D. Anisotropy of age-related toughness loss in human cortical bone: A finite element study. J. Biomech. 40, 1606-1614 (2007).

22. Ural, A. Cohesive modeling of bone fracture at multiple scales. Procedia Eng. 10, $2827-$ $2832(2011)$

23. Leng, X., Chen, X., Deng, X., Sutton, M. A. \& Lessner, S. M. Modeling of Experimental Atherosclerotic Plaque Delamination. Ann. Biomed. Eng. 43, 2838-2851 (2015).

24. Sibinga, N. E. et al. Collagen VIII is expressed by vascular smooth muscle cells in response to vascular injury. Circ. Res. 80, 532-541 (1997).

25. Plenz, G., Dorszewski, A., Breithardt, G. \& Robenek, H. Expression of type VIII collagen after cholesterol diet and injury in the rabbit model of atherosclerosis. Arterioscler. Thromb. Vasc. Biol. 19, 1201-1209 (1999).

26. Wang, Y., Johnson, J. A., Spinale, F. G., Sutton, M. A. \& Lessner, S. M. Quantitative Measurement of Dissection Resistance in Intimal and Medial Layers of Human Coronary Arteries. Exp. Mech. 54, 677-683 (2014).

27. Analysis User's Manual Version 6.13. ABAQUS. (Dassault Systemes Corp, 2013).

28. Chen, X., Deng, X. \& Sutton, M. A. Simulation of stable tearing crack growth events using the CZM approach with an explicit solver. Finite Elem. Anal. Des. 81, 32-37 (2014). 
29. Pagani, M. \& Perego, U. Explicit dynamics simulation of blade cutting of thin elastoplastic shells using 'directional' cohesive elements in solid-shell finite element models. Comput. Methods Appl. Mech. Eng. 285, 515-541 (2015).

30. Gregersen, H., Zhao, J., Lu, X., Zhou, J. \& Falk, E. Remodelling of the zero-stress state and residual strains in apoE-deficient mouse aorta. Biorheology 44, 75-89 (2007).

31. Hill, M. R., Duan, X., Gibson, G. A., Watkins, S. \& Robertson, A. M. A theoretical and nondestructive experimental approach for direct inclusion of measured collagen orientation and recruitment into mechanical models of the artery wall. J. Biomech. 45, 762-771 (2012).

32. Yosibash, Z. \& Priel, E. Artery active mechanical response: High order finite element implementation and investigation. Comput. Methods Appl. Mech. Eng. 237-240, 51-66 (2012).

33. Chai, C.-K. et al. Local axial compressive mechanical properties of human carotid atherosclerotic plaques-characterisation by indentation test and inverse finite element analysis. J. Biomech. 46, 1759-1766 (2013).

34. Le Floc'h, S. et al. Vulnerable atherosclerotic plaque elasticity reconstruction based on a segmentation-driven optimization procedure using strain measurements: theoretical framework. IEEE Trans. Med. Imaging 28, 1126-1137 (2009).

35. Merei, B., Avril, S., Badel, P., Sutton, M. A. \& Lessner, S. M. Numerical study of delamination through human aortic media using cohesive elements and two different material laws: linear elastic and hyperelastic. in (Mira Digital Publishing, 2012).

36. Teng, Z. et al. Material properties of components in human carotid atherosclerotic plaques: A uniaxial extension study. Acta Biomater. 10, 5055-5063 (2014). 
37. Song, K., Davila, C. G. \& Rose, C. A. Guidelines and Parameter Selection for the Simulation of Progressive Delamination. (2008).

38. Lopes, J. et al. Type VIII Collagen Mediates Vessel Wall Remodeling after Arterial Injury and Fibrous Cap Formation in Atherosclerosis. Am. J. Pathol. 182, 2241-2253 (2013).

39. Untaroiu, C. D., Lu, Y.-C., Siripurapu, S. K. \& Kemper, A. R. Modeling the biomechanical and injury response of human liver parenchyma under tensile loading. J. Mech. Behav. Biomed. Mater. 41, 280-291 (2015).

40. Elices, M., Guinea, G. V., Gómez, J. \& Planas, J. The cohesive zone model: advantages, limitations and challenges. Eng. Fract. Mech. 69, 137-163 (2002).

41. Chandra, N., Li, H., Shet, C. \& Ghonem, H. Some issues in the application of cohesive zone models for metal-ceramic interfaces. Int. J. Solids Struct. 39, 2827-2855 (2002).

42. Kyoungsoo Park, G. H. P. Adaptive mesh refinement and coarsening for cohesive zone modeling of dynamic fracture. Int. J. Numer. Methods Eng. 92, 1-35 (2012).

43. Wang, J. T. Investigating Some Technical Issues on Cohesive Zone Modeling of Fracture. $J$. Eng. Mater. Technol. 135, $011003-011003$ (2012).

44. Assoul, N., Flaud, P., Chaouat, M., Letourneur, D. \& Bataille, I. Mechanical properties of rat thoracic and abdominal aortas. J. Biomech. 41, 2227-2236 (2008).

45. Smith, E. B. \& Slater, R. S. The microdissection of large atherosclerotic plaques to give morphologically and topographically defined fractions for analysis: Part 1. The lipids in the isolated fractions. Atherosclerosis 15, 37-56 (1972).

46. Small, D. M. George Lyman Duff memorial lecture. Progression and regression of atherosclerotic lesions. Insights from lipid physical biochemistry. Arterioscler. Dallas Tex $\mathbf{8}$, 103-129 (1988). 
47. Akyildiz, A. C., Speelman, L. \& Gijsen, F. J. H. Mechanical properties of human atherosclerotic intima tissue. J. Biomech. 47, 773-783 (2014).

48. Kanai, H., Hasegawa, H., Ichiki, M., Tezuka, F. \& Koiwa, Y. Elasticity Imaging of Atheroma With Transcutaneous Ultrasound Preliminary Study. Circulation 107, 3018-3021 (2003). 


\section{List of Tables}

Table 1: Number of plaques and cycles obtained from each mouse group

Table 2: Plaque Geometry: (Lp) Plaque Length, (Wp) Plaque Width and (Hp) Plaque Height

Table 3: Statistical parameters for energy release rate, stiffness, and failure load values for ApoE-/- and ApoE-/- Col8-/mice. 


\begin{tabular}{|c|c|c|c|}
\hline & Mouse ID & Plaque ID & Total Cycles \\
\hline \multirow{5}{*}{ ApoE ${ }^{-/-}$} & 124 & P1 & 7 \\
\hline & 145 & $\mathrm{P} 1$ & 2 \\
\hline & 158 & P1 & 2 \\
\hline & \multirow[t]{2}{*}{161} & P1 & 2 \\
\hline & & $\mathrm{P} 2$ & 4 \\
\hline Total & 4 & 5 & 17 \\
\hline \multirow{9}{*}{$\operatorname{ApoE}^{-/-} \mathrm{Col}^{-/-}$} & 150 & $\mathrm{P} 1$ & 1 \\
\hline & 151 & $\mathrm{P} 1$ & 1 \\
\hline & 152 & $\mathrm{P} 1$ & 3 \\
\hline & 157 & $\mathrm{P} 1$ & 2 \\
\hline & 173 & $\mathrm{P} 1$ & 2 \\
\hline & \multirow[t]{3}{*}{174} & $\mathrm{P} 1$ & 1 \\
\hline & & $\mathrm{P} 2$ & 5 \\
\hline & & P3 & 4 \\
\hline & 175 & $\mathrm{P} 1$ & 3 \\
\hline Total & 7 & 9 & 22 \\
\hline
\end{tabular}

Table 4: Number of plaques and cycles obtained from each mouse group

\begin{tabular}{|l|l|l|l|l|l|}
\hline & Mouse & Plaque & Lp (mm) & Wp (mm) & Hp (mm) \\
\hline ApoE $^{-/}$ & 124 & P1 & 4.65 & 0.4 & 0.5 \\
\hline
\end{tabular}




\begin{tabular}{|c|c|c|c|c|c|}
\hline & 145 & $\mathrm{P} 1$ & 1.8 & 0.45 & 0.18 \\
\hline & 158 & P1 & 3.5 & 0.6 & 0.32 \\
\hline & 161 & $\mathrm{P} 1$ & 3.2 & 0.75 & 0.14 \\
\hline & & $\mathrm{P} 2$ & 3.2 & 0.9 & 0.30 \\
\hline $\mathbf{A p o E}^{-/-}$ & 150 & $\mathrm{P} 1$ & 2.8 & 0.94 & 0.17 \\
\hline $\mathrm{Col}^{-/-}$ & 151 & $\mathrm{P} 1$ & 4 & 0.8 & 0.28 \\
\hline & 152 & P1 & 2.8 & 0.9 & 0.3 \\
\hline & 157 & P1 & 3.8 & 0.4 & 0.39 \\
\hline & 173 & P1 & 3.6 & 0.7 & 0.13 \\
\hline & 174 & P1 & 2.5 & 0.74 & 0.18 \\
\hline & & $\mathrm{P} 2$ & 4 & 0.47 & 0.11 \\
\hline & & P3 & 2.6 & 0.75 & 0.14 \\
\hline & 175 & P1 & 2.8 & 0.5 & 0.12 \\
\hline
\end{tabular}

Table 5: Plaque Geometry: (Lp) Plaque Length, (Wp) Plaque Width and (Hp) Plaque Height 


\begin{tabular}{|l|c|c|}
\cline { 2 - 3 } \multicolumn{1}{c|}{} & \multicolumn{2}{c|}{ G [N/mm] } \\
\cline { 2 - 3 } \multicolumn{1}{c|}{} & $\mathbf{A p o E}$ & $\mathbf{A p o E}^{-/-} \mathbf{C o l 8}^{-/-}$ \\
\hline Average values & 0.015 & 0.016 \\
\hline Median & 0.01 & 0.01 \\
\hline Standard deviation & 0.011 & 0.018 \\
\hline First quartile & 0.008 & 0.0075 \\
\hline Third quartile & 0.018 & 0.015 \\
\hline
\end{tabular}

Table 6: Statistical parameters for energy release rate, stiffness, and failure load values for ApoE-/- and ApoE-/- Col8-/mice. 


\section{List of figures}

Figure 1: Schematic of the experimental setup; (a): the Bose machine prescribes a displacement (actuator) and records the resulting force (load cell). The micro-clamps are attached to the actuator and grip the tip of the plaque (b) Schematic of delamination process

Figure 2: A representative image of the raw force and displacement data. The area under the load-displacement curve represents the energy released during one delamination cycle. The linear region depicted is used to determine the plaque stiffness for each cycle

Figure 3: The estimated $\Delta \mathrm{A}$ for one cycle, outlined by yellow (top view)

Figure 4 : Schematic representation of the plaque model and the underlying aorta. (a): $\mathrm{Lm}$ represents the aortic media length, Lp the plaque length, Wm the medial width, Wp the plaque width, Hm the medial height and Hp the maximum plaque height (Lm not shown to scale); (b): 2D representation of the atherosclerotic plaque (green) attached by cohesive elements to the underlying aorta (blue), lying on the gray rigid surface (S). The bottom edge of $S$, the left \& right edges of $(A+S)$, and the top left edge of A were clamped to simulate experimental testing conditions. The reference point represents the master node where displacement boundary conditions were applied.

Figure 5: Simulation of the peeling test at 4 different times throughout the test. (a): the initial position before the application of the boundary condition, (b) vertical displacement to move the free arm of the plaque, (c) application of horizontal displacement to simulate the delamination process, (d) evolution of the delamination

Figure 6: Traction/separation curve for Bilinear Cohesive Zone model

Figure 2: Variation of cost function values with respect to $\mathrm{C}_{10}$, with $\mathrm{T}_{0}=0.05-0.10 \mathrm{MPa}$ for the $\mathrm{Sample}^{173 P 1} \mathrm{ApoE}^{-/-} \mathrm{Col}^{-/-}$

Figure 8: Force-displacement curve obtained after simulation for 1 sample. The curve consists of three segments: 1 represents the deformation of the attached peel arm of the plaque, 2 represents the separation phase where the cohesive elements are deleted to simulate the separation, and 3 represents the unloading phase. Segment 2 displays serrations related to the deletion of cohesive elements

Figure 9: Experimental vs simulated force-displacement curves and strain vs kinetic energy for the first delamination cycles from four ApoE-/- mice

Figure 10: Experimental vs simulated force-displacement curves and strain vs kinetic energy for the first delamination cycles from four ApoE -/- Col8-/- mice

Figure 3: Histograms of average identified values and standard deviations for (a) $G$, (b) $T_{0}$, and (c) $C_{10}$

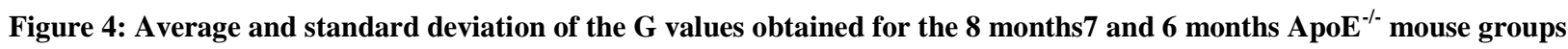




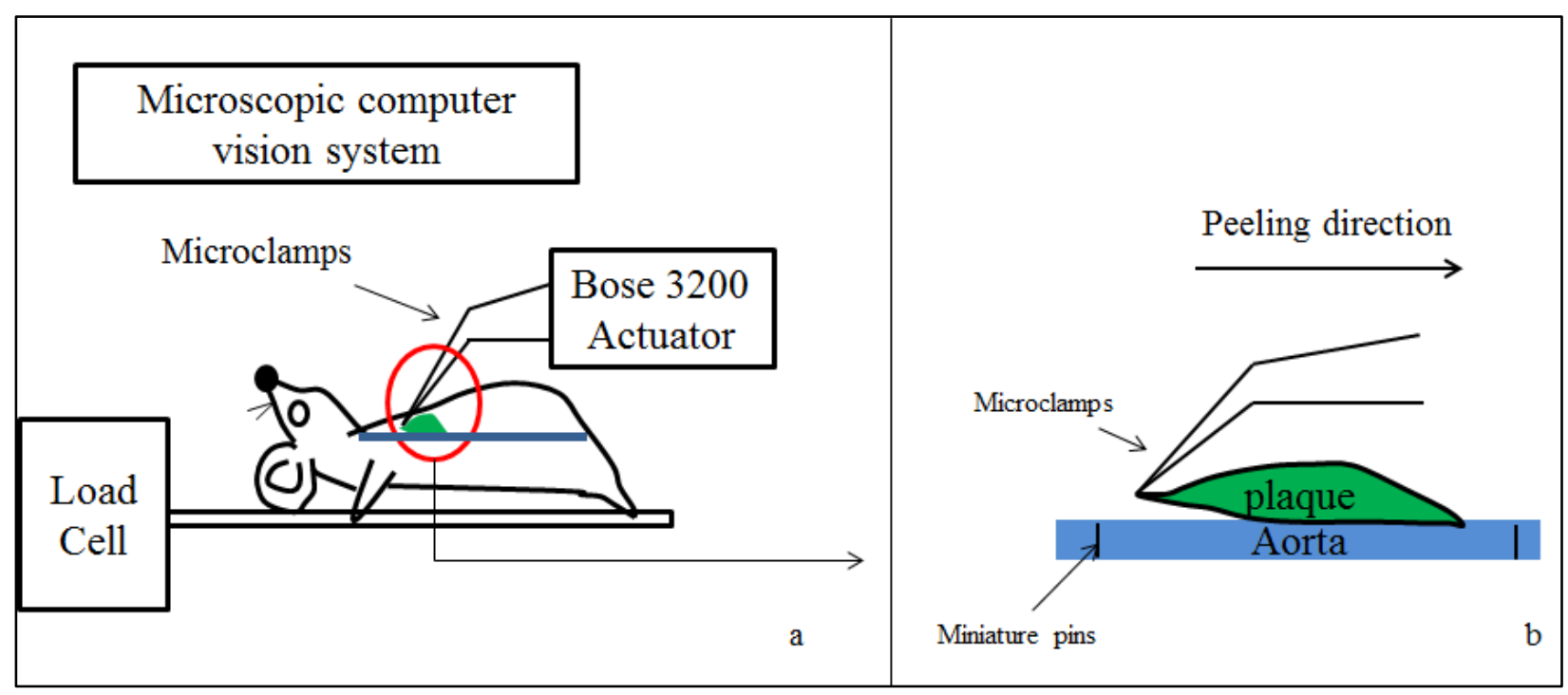

Figure 5: Schematic of the experimental setup; (a): the Bose machine prescribes a displacement (actuator) and records the resulting force (load cell). The micro-clamps are attached to the actuator and grip the tip of the plaque (b) Schematic of delamination process 


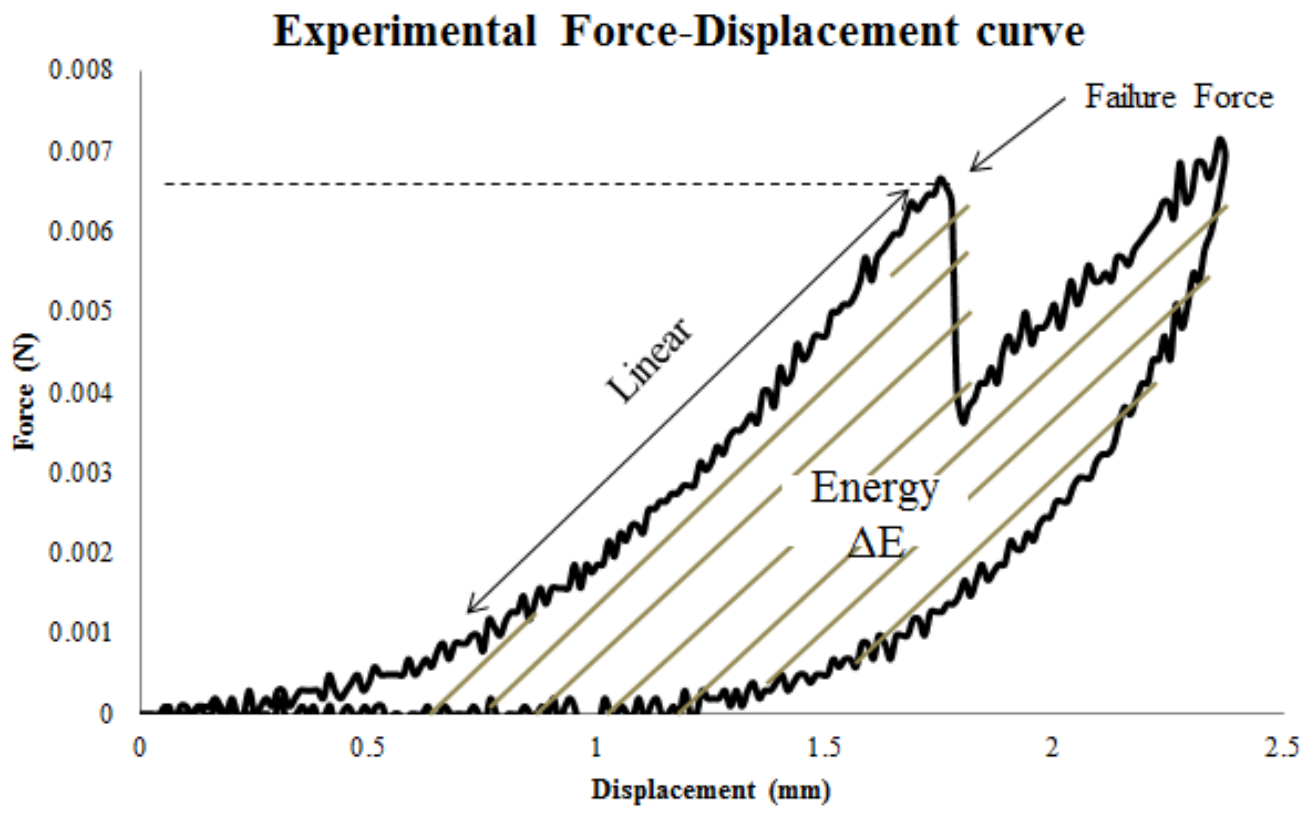

Figure 6: A representative image of the raw force and displacement data. The area under the load-displacement curve represents the energy released during one delamination cycle. The linear region depicted is used to determine the plaque stiffness for each cycle 


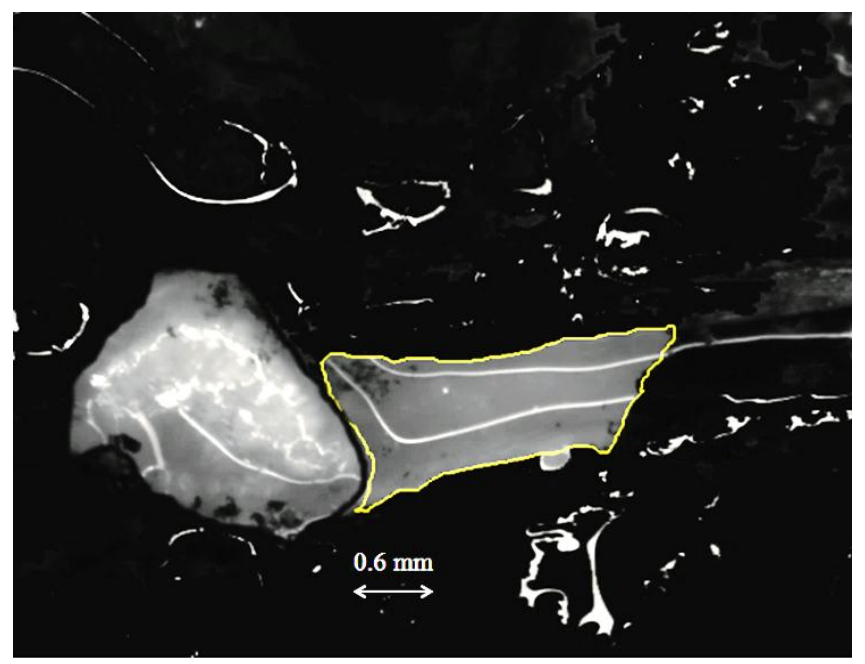

Figure 7: The estimated $\Delta \mathrm{A}$ for one cycle, outlined by yellow (top view) 


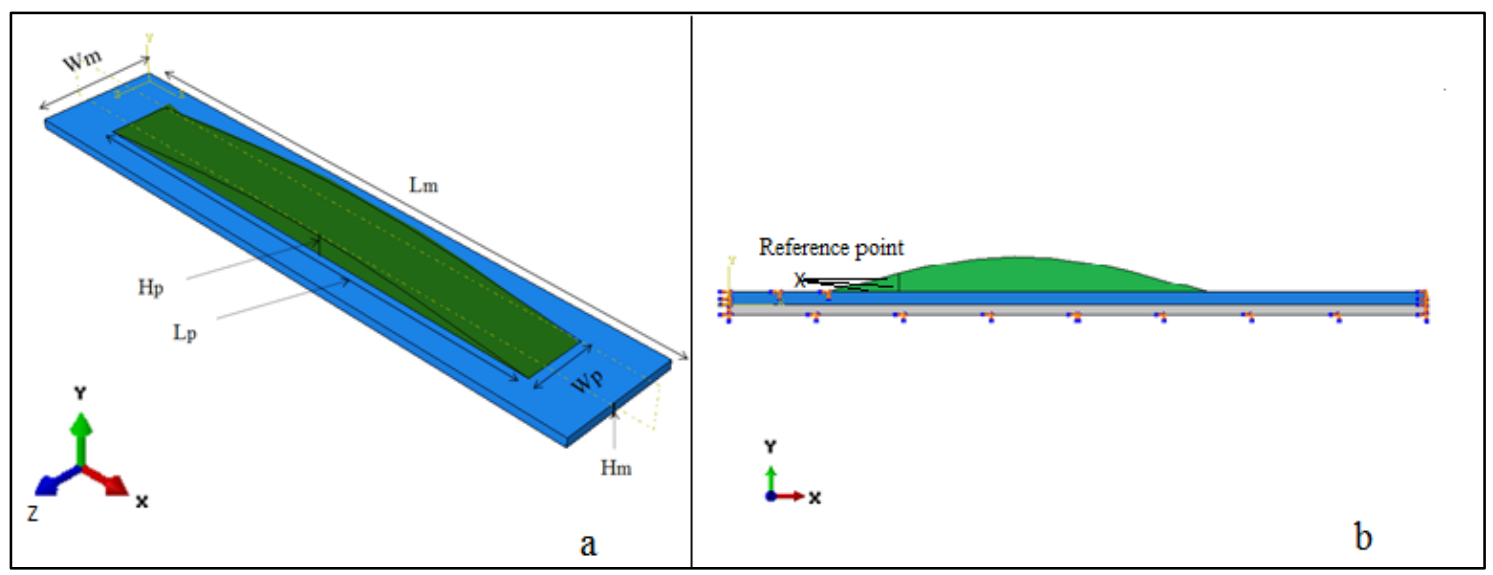

Figure 8 : Schematic representation of the plaque model and the underlying aorta. (a): Lm represents the aortic media length, Lp the plaque length, Wm the medial width, Wp the plaque width, Hm the medial height and Hp the maximum plaque height (Lm not shown to scale); (b): 2D representation of the atherosclerotic plaque (green) attached by cohesive elements to the underlying aorta (blue), lying on the gray rigid surface $(S)$. The bottom edge of $S$, the left $\&$ right edges of

$(A+S)$, and the top left edge of $A$ were clamped to simulate experimental testing conditions. The reference point represents the master node where displacement boundary conditions were applied. 


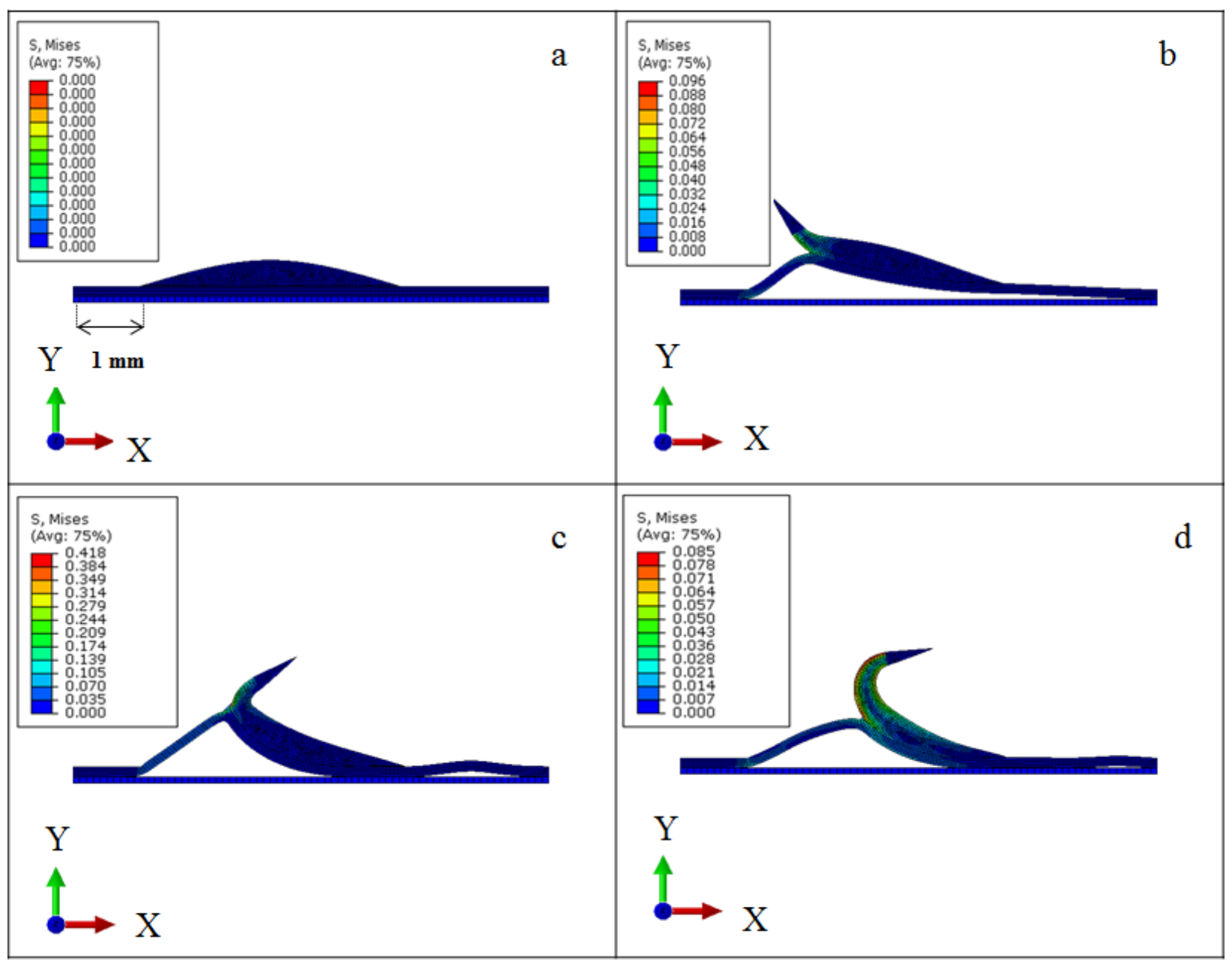

Figure 9: Simulation of the peeling test at 4 different times throughout the test. (a): the initial position before the application of the boundary condition, (b) vertical displacement to move the free arm of the plaque, (c) application of horizontal displacement to simulate the delamination process, (d) evolution of the delamination 


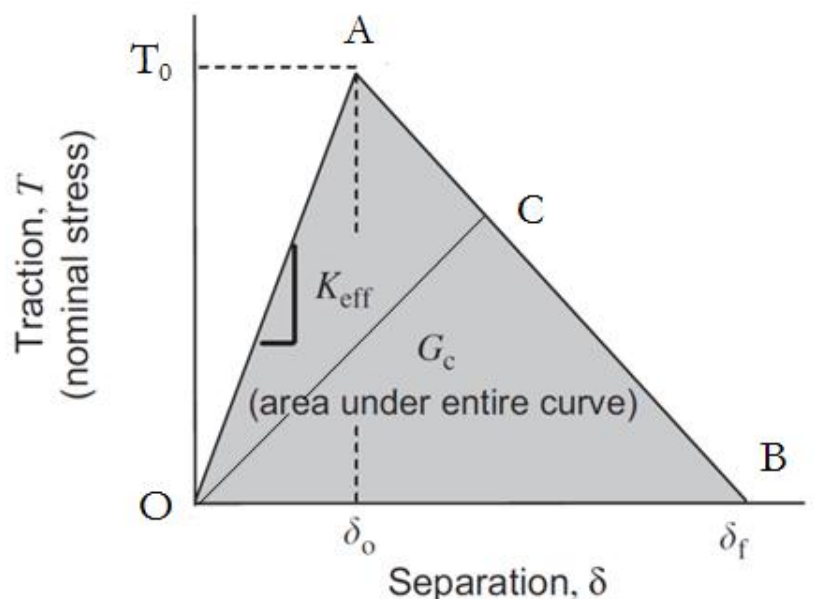

Figure 10: Traction/separation curve for Bilinear Cohesive Zone model 


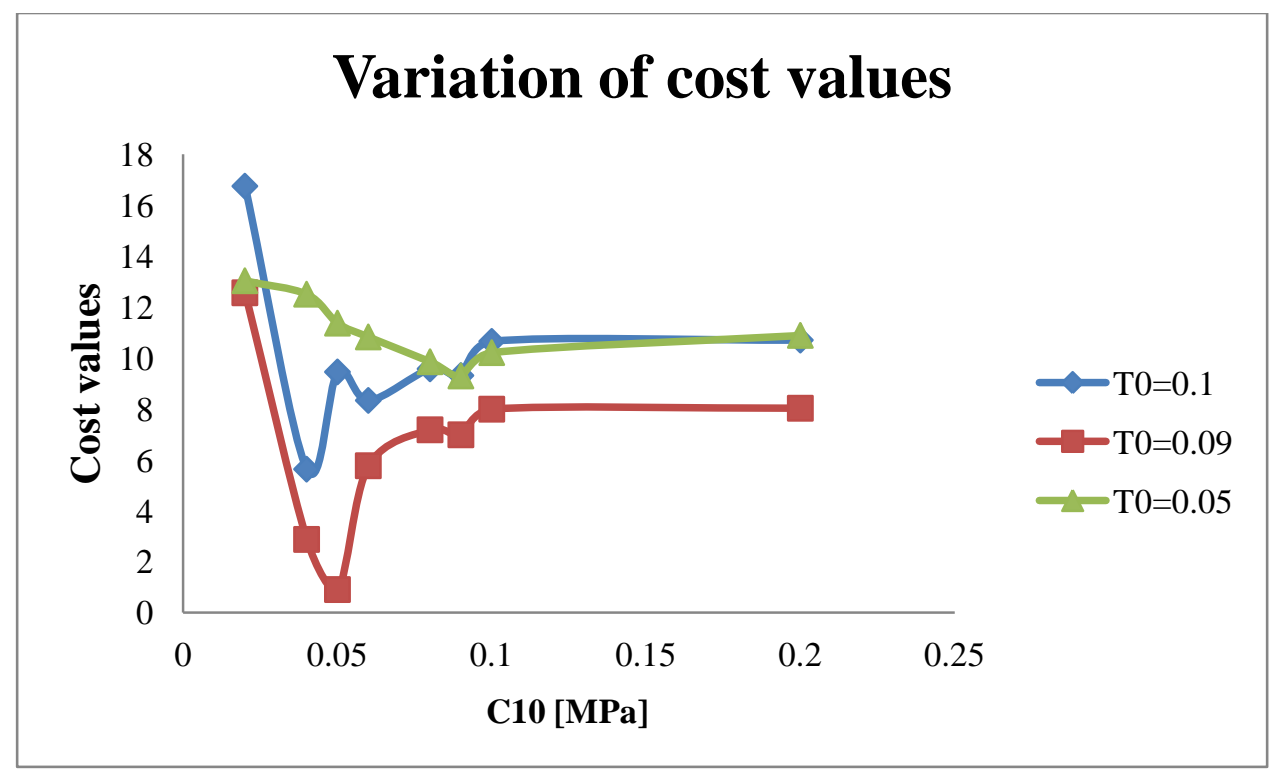

Figure 11: Variation of cost function values with respect to $C_{10}$, with $T_{0}=0.05-0.10 \mathrm{MPa}$ for the sample $173 \mathrm{P} 1 \mathrm{ApoE} \mathrm{E}^{-/-}$ $\mathrm{Col}^{-/-}$ 


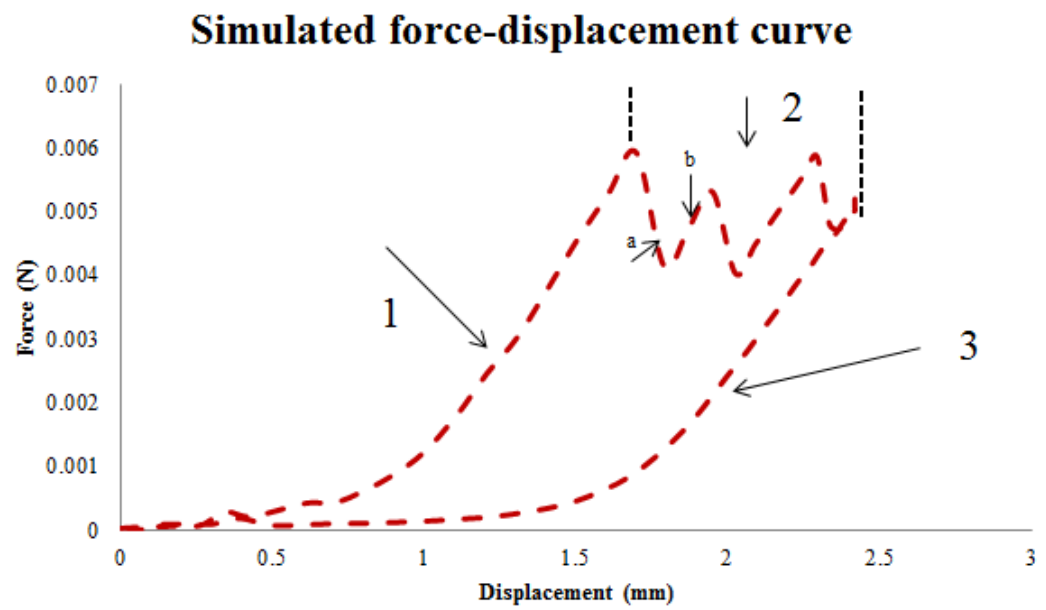

Figure 12: Force-displacement curve obtained after simulation for 1 sample. The curve consists of three segments: 1 represents the deformation of the attached peel arm of the plaque, 2 represents the separation phase where the cohesive elements are deleted to simulate the separation, and 3 represents the unloading phase. Segment 2 displays serrations related to the deletion of cohesive elements 


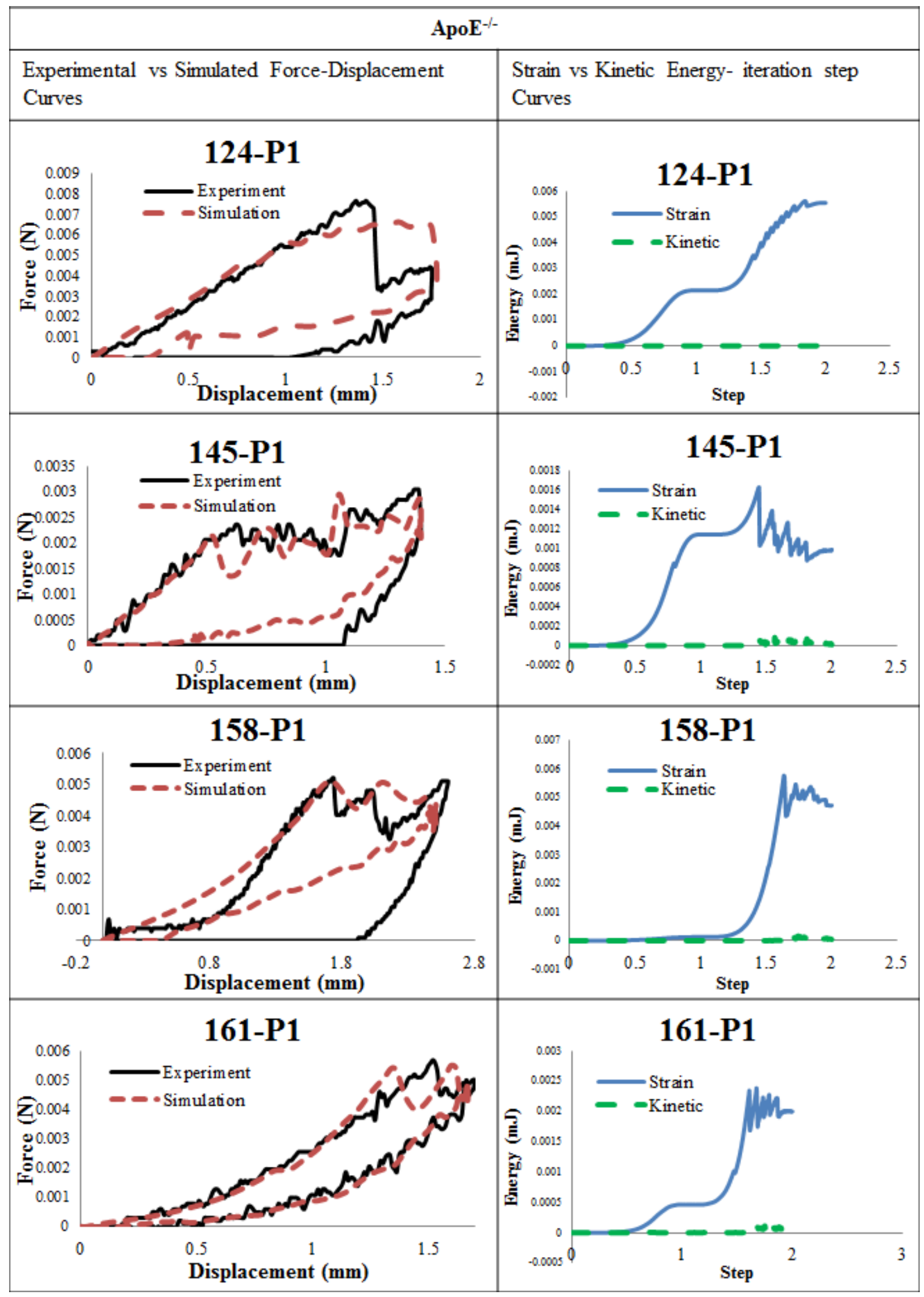

Figure 13: Experimental vs simulated force-displacement curves and strain vs kinetic energy for the first delamination cycles from four $\mathrm{ApoE}^{-/-}$mice 


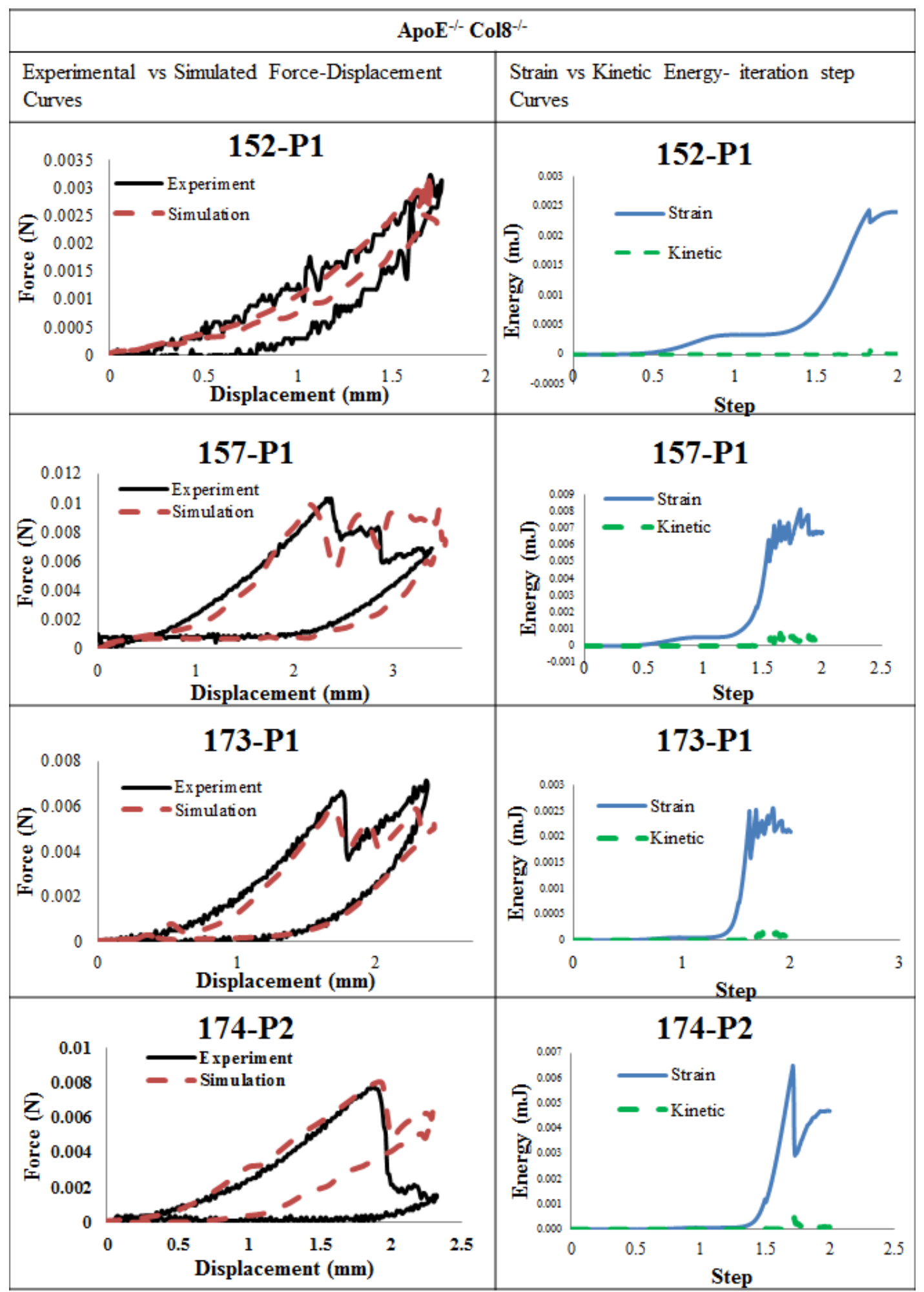

Figure 14: Experimental vs simulated force-displacement curves and strain vs kinetic energy for the first delamination cycles from four ApoE -/- Col8-/- mice 


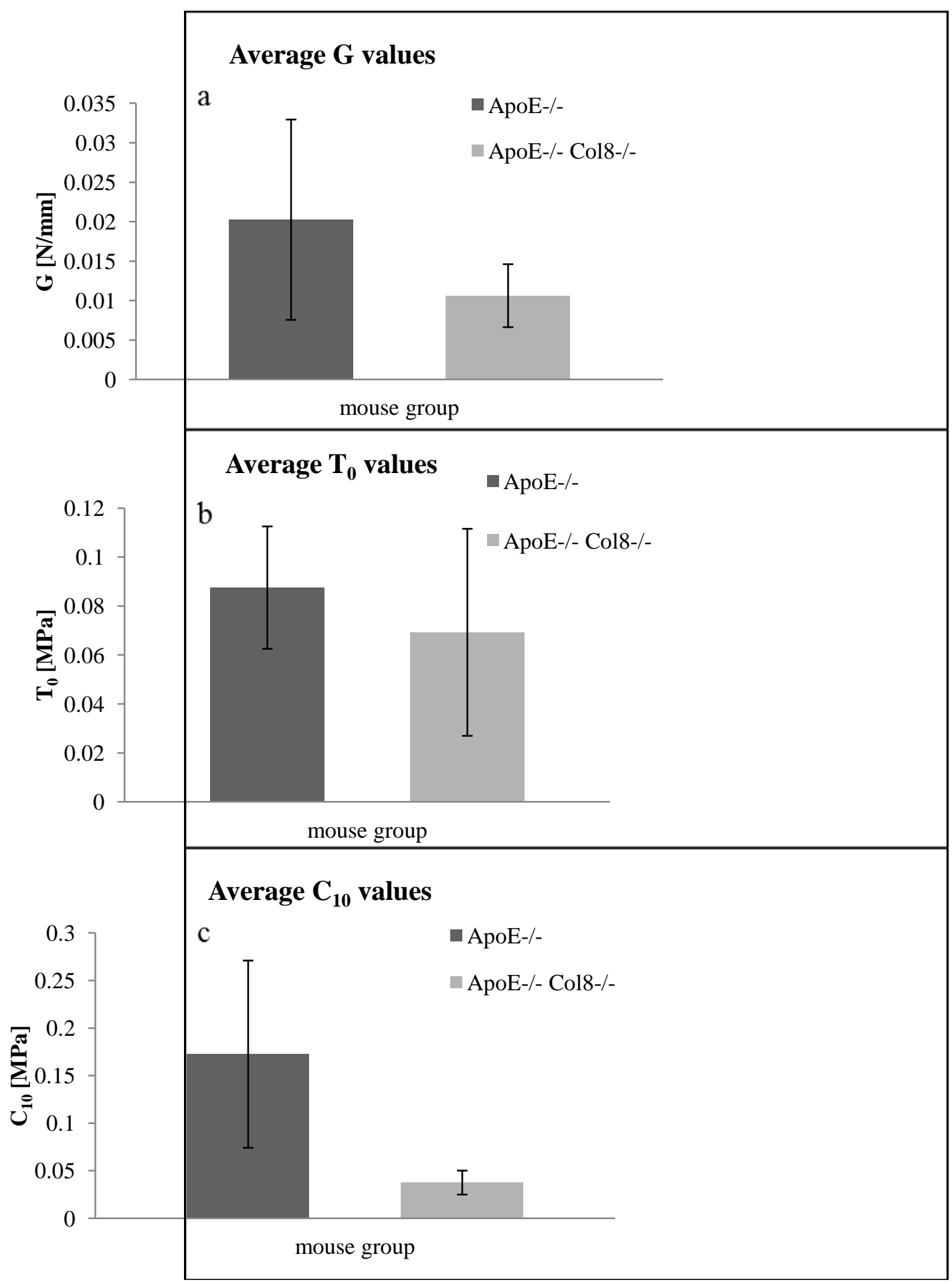

Figure 15: Histograms of average identified values and standard deviations for (a) $G$, (b) $\mathbf{T}_{0}$, and (c) $\mathbf{C}_{10}$ 


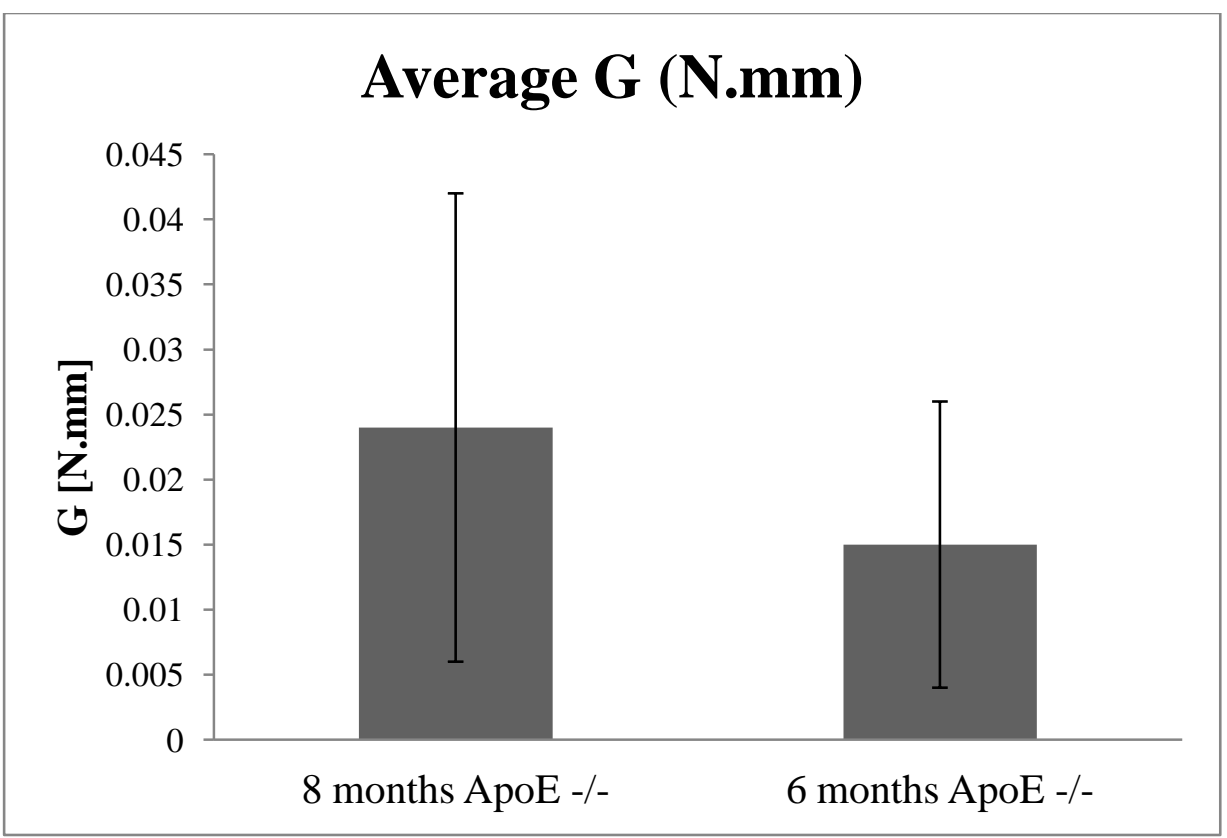

Figure 16: Average and standard deviation of the $G$ values obtained for the 8 months7 and 6 months ApoE ${ }^{-/-}$mouse $^{-1}$ groups 